\title{
Reducing False-Positive Results With Fourth-Generation HIV Testing at a Veterans Affairs Medical Center
}

\author{
Jeffrey Petersen, MD; Maria Monteiro, MT; Sharvari Dalal, MD; and Darshana Jhala, MD
}

\begin{abstract}
Background: In 2006, the Centers for Disease Control and Prevention issued guidelines advocating routine HIV screening for all patients. However, false-positive results are a potential patient care threat for low-risk populations even with accurate screening assays. A reduction in HIV falsepositive screening results can potentially be seen by switching from the third-generation to a more sensitive and specific fourth-generation screening assay.

Methods: We studied the impact on the false-positive screening rate of a change to a fourth-generation assay at a regional US Department of Veteran Affairs Medical Center. HIV screening tests performed by the laboratory from March 1, 2016 to February 28,2017 , prior to implementation of the new assay were compared with fourth-generation HIV screening tests performed from March 1, 2017 to February 28, 2018.
\end{abstract}

Results: Of 7,516 third-generation HIV screening tests reviewed, 52 were reactive on the screening assay; 24 were true positives, 28 were false positives. The following year 7,802 fourth-generation HIV screening tests were performed and 23 were reactive on the screening assay; 16 were true positives and 7 were false positives. The positive predictive value for the third-generation test was $46 \%$ and $70 \%$ for the fourth-generation test.

Conclusions: There were fewer false-positive results with testing with the more specific fourth- vs third-generation assay $(0.09 \%$ vs $0.37 \%$, respectively), which was statistically significant $(P=.002)$. This reduction in false-positive screening would reduce the laboratory workload and would save an estimated $\$ 3,875$ yearly and reduce the adverse effects of false-positive screening results for patients.
Author affiliations can be found at the end of the article.

Correspondence: Jeffrey Petersen (jeffrey.petersen@va.gov)

Fed Pract. 2021;38(5). Published online May 12. doi: $10.12788 /$ fp. 0125

\begin{abstract}
ver since the first clinical reports of pa- tients with AIDS in 1981, there have been improvements both in the knowledge base of the pathogenesis of HIV in causing AIDS as well as a progressive refinement in the test methodologies used to diagnose this illness. ${ }^{1-3}$ Given that there are both public health and clinical benefits in earlier diagnosis and treatment of patients with available antiretroviral therapies, universal screening with opt-out consent has been a standard of practice recommendation by the Centers of Disease Control and Prevention (CDC) since 2006; universal screening with opt-out consent also has been recommended by the US Preventative Task Force and has been widely implemented. ${ }^{4-7}$
\end{abstract}

\section{HIV SCREENING}

While HIV screening assays have evolved to be accurate with very high sensitivities and specificities, false-positive results are a significant issue both currently and historically.-16 The use of an HIV assay on a low prevalence population predictably reduces the positive predictive value (PPV) of even an otherwise accurate assay. ${ }^{8-23}$ In light of this, laboratory HIV testing algorithms include confirmatory testing to increase the likelihood that the correct diagnosis is being rendered.

The fourth-generation assay has been shown to be more sensitive and specific compared with that of the thirdgeneration assay due to the addition of detection of p24 antigen and the refinement of the antigenic targets for the antibody detection. ${ }^{6,8,11-13,18-20,22}$ Due to these improvements, in the general population, increased sensitivity/specificity with a reduction in both false positives and false negatives have been reported.

It has been observed in the nonveteran population that switching from the older thirdgeneration to a more sensitive and specific fourth-generation HIV screening assay has reduced the false-positive screening rate. ${ }^{18,19,22}$ For instance, Muthukumar and colleagues demonstrated a false-positive rate of only 2 out of 99 (2\%) tested specimens for the fourth-generation ARCHITECT HIV Ag/Ab Combo assay vs 9 out of 99 tested specimens (9\%) for the third-generation ADVIA Centaur HIV 1/O/2 Enhanced assay. ${ }^{18}$ In addition, it has been noted that fourth-generation HIV screening assays can reduce the window period by detecting HIV infection sooner after initial acute infection. ${ }^{19}$ Mitchell and colleagues demonstrated even highly specific fourth-generation HIV assays with specificities estimated at $99.7 \%$ can have PPVs as low as $25.0 \%$ if used in a population of low HIV prevalence (such as a $0.1 \%$ prevalence 
population) ${ }^{19}$ However, the veteran population has been documented to differ significantly on a number of population variables, including severity of disease and susceptibility to infections, and as a result extrapolation of these data from the general population may be limited. ${ }^{24-26}$ To our knowledge, this article represents the first study directly examining the reduction in false-positive results with the switch to a fourth-generation HIV generation assay from a third-generation assay for the veteran patient population at a regional US Department of Veterans Affairs (VA) medical center (VAMC). ${ }^{8,11}$

\section{METHODS}

Quality assurance documents on test volume were retrospectively reviewed to obtain the number of HIV screening tests that were performed by the laboratory at the Corporal Michael J. Crescenz VAMC (CMJCVAMC) in Philadelphia, Pennsylvania, between March 1, 2016 and February 28,2017 , prior to implementation of the fourth-generation assay. The study also include results from the first year of use of the fourth-generation assay (March 1, 2017 to February 28, 2018). In addition, paper quality assurance records of all positive screening results during those periods were reviewed and manually counted for the abstract presentation of these data.

For assurance of accuracy, a search of all HIV testing assays using Veterans Health Information Systems and Technology Architecture and FileMan also was performed, and the results were compared to records in the Computerized Patient Record System (CPRS). Any discrepancies in the numbers of test results generated by both searches were investigated, and data for the manuscript were derived from records associating tests with particular patients. Only results from patient samples were considered for the electronic search. Quality samples that did not correspond to a true patient as identified in CPRS or same time patient sample duplicates were excluded from the calculations. Basic demographic data (age, ethnicity, and gender) were obtained from this FileMan search. The third-generation assay was the Ortho-Clinical Diagnostics Vitros, and the fourth-generation assay was the Abbott Architect.
TABLE Patient Demographics

\begin{tabular}{lcc} 
& \multicolumn{2}{c}{ HIV Test Generation, No. } \\
\cline { 2 - 3 } Characteristics & $\begin{array}{c}\text { Third } \\
(\mathbf{n}=\mathbf{7 , 5 1 6 )}\end{array}$ & $\begin{array}{c}\text { Fourth } \\
(\mathbf{n}=\mathbf{7}, 802)\end{array}$ \\
\hline Age, y & & \\
$20-29$ & 98 & 179 \\
$30-39$ & 1,097 & 1,181 \\
$40-49$ & 751 & 836 \\
$50-59$ & 1,169 & 1,217 \\
$60-69$ & 1,838 & 1,784 \\
$70-79$ & 1,292 & 1,416 \\
$80-89$ & 129 & 167 \\
$90-99$ & 28 & 29 \\
Not available & 1,114 & 993 \\
\hline Ethnicity & & \\
American Indian or Alaska Native & 37 & 51 \\
Asian American & 74 & 76 \\
African American & 3,220 & 3,189 \\
Declined to state/unknown & 278 & 298 \\
Native Hawaiian or other Pacific Islander & 37 & 46 \\
White & 2,756 & 3,149 \\
Not available & 1,114 & 993 \\
\hline Gender & & \\
Male & & 5,971 \\
Female & 838 \\
Not available & 7639 & 993 \\
\end{tabular}

To interpret the true HIV result of each sample with a reactive or positive screening result, the CDC laboratory HIV testing algorithm was followed and reviewed with a clinical pathologist or microbiologist director. ${ }^{12,13}$ All specimens interpreted as HIV positive by the pathologist or microbiologist director were discussed with the clinical health care provider at the time of the test with results added to CPRS after all testing was complete and discussions had taken place. All initially reactive specimens (confirmed with retesting in duplicate on the screening platform with at least 1 repeat reactive result) were further tested with the Bio-Rad Geenius HIV 1/2 Supplemental Assay, which screens for both HIV-1 and HIV-2 antibodies. Specimens with reactive results by this supplemental assay were interpreted as positive for HIV based on the CDC laboratory HIV testing algorithm. Specimens with negative or indeterminant results by the supplemental assay then underwent HIV-1 nucleic acid testing (NAT) using the Roche Diagnostics COBAS AmpliPrep/COBAS TaqMan HIV-1 Test v2.0. Specimens with viral load detected on NAT were positive for HIV infection, while specimens with viral load not detected on 
FIGURE 1 Positive HIV Assay Results

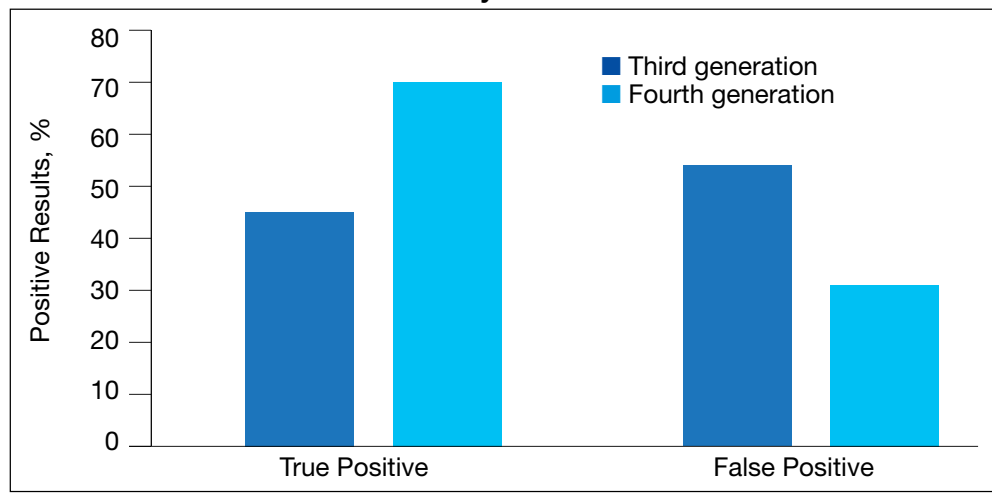

NAT testing were interpreted as negative for HIV-1 infection. Although there were no HIV-2 positive or indeterminant specimens during the study period, HIV-2 reactivity also would have been interpreted per the CDC laboratory HIV testing algorithm. Specimens with inadequate volume to complete all testing steps would be interpreted as indeterminant for HIV with request for additional specimen to complete testing. All testing platforms used for HIV testing in the laboratory had been properly validated prior to use.

The number of false positives and indeterminant results was tabulated in Microsoft Excel by month throughout the study period alongside the total number of HIV screening tests performed. Statistical analyses to verify statistical significance was performed by 1-tailed homoscedastic $t$ test calculation using Excel.

\section{RESULTS}

From March 1, 2016 to February 28, $2017,7,516$ specimens were screened for HIV, using the third-generation assay, and 52 specimens tested positive for HIV. On further review of these reactive specimens per the CDC laboratory testing algorithm, 24 tests were true positive and 28 were false positives with a PPV of $46 \%$ (24/52) (Figure 1).

From March 1, 2017 to February 28, 2018, 7,802 specimens were screened for HIV using a fourth-generation assay and 23 tested positive for HIV. On further review of these reactive specimens per the CDC laboratory testing algorithm, 16 were true positive and 7 were false positives with a PPV of $70 \%(16 / 23)$.

The fourth-generation assay was more specific when compared with the third- generation assay $(0.09 \%$ vs $0.37 \%$, respectively) with a $75.7 \%$ decrease in the false-positivity rate after the implementation of fourth-generation testing. The decreased number of false-positive test results per month with the fourth-generation test implementation was statistically significant $(P=.002)$. The mean (SD) number of falsepositive test results for the third-generation assay was 2.3 (1.7) per month, while the fourth-generation assay only had a mean (SD) of 0.58 (0.9) false positives monthly. The decrease in the percentage of false positives per month with the implementation of the fourth-generation assay also was statistically significant $(P=.002)$ (Figure 2$)$.

For population-based reference of the tested population at CMJCVAMC, there was a FileMan search for basic demographic data of patients for the HIV specimens screened by the third- or fourth-generation test (Table). For the population tested by the third-generation assay, 1,114 out of the 7,516 total tested population did not have readily available demographic information by the FileMan search as the specimens originated outside of the facility. For 6,402 of 7,516 patients tested by the third-generation assay with demographic information, the age ranged from 25 to 97 years with a mean of 57 years. This population of 6,402 was $88 \%$ male $(\mathrm{n}=5,639), 50 \%$ African American $(\mathrm{n}=3,220)$ and $43 \%$ White $(\mathrm{n}=2,756)$. For the population tested by the fourthgeneration assay, 993 of 7,802 total tested population did not have readily available demographic information by the FileMan search as the specimens originated outside of the facility. For the 6,809 of $7,802 \mathrm{pa}$ tients tested by the fourth-generation assay with demographic information, the age ranged from 24 to 97 years with a mean age of 56 years. This population was $88 \%$ male $(\mathrm{n}=5,971), 47 \%$ African American ( $\mathrm{n}=$ $3,189)$, and $46 \%$ White $(n=3,149)$.

\section{DISCUSSION}

Current practice guidelines from the CDC and the US Preventive Services Task Force recommend universal screening of the population for HIV infection..$^{5,6}$ As the general population to be screened would normally have a low prevalence of HIV infection, the risk of a false positive on the initial screen 
is significant. ${ }^{17}$ Indeed, the CMJCVAMC experience has been that with the thirdgeneration screening assay, the number of false-positive test results outnumbered the number of true-positive test results. Even with the fourth-generation assay, approximately one-third of the results were false positives. These results are similar to those observed in studies involving nonveteran populations in which the implementation of a fourth-generation screening assay led to significantly fewer false-positive results. ${ }^{18}$

For laboratories that do not follows CDC testing algorithm guidelines, each falsepositive screening result represents a potential opportunity for a HIV misdiagnosis. Even in laboratories with proper procedures in place, false-positive results have consequences for the patients and for the costeffectiveness of laboratory operations..$^{9-11,18}$ As per CDC HIV testing guidelines, all positive screening results should be retested, which leads to additional use of technologist time and reagents. After this additional testing is performed and reviewed appropriately, only then can an appropriate final laboratory diagnosis be rendered that meets the standard of laboratory care.

\section{Cost Savings}

As observed at CMJCVAMC, the use of a fourth-generation assay with increased sensitivity/specificity led to a reduction in these false-positive results, which improved laboratory efficiency and avoided wasted resources for confirmatory tests. ${ }^{11,18}$ Cost savings at CMJCVAMC from the implementation of the fourth-generation assay would include technologist time and reagent cost. Generalizable technologist time costs at any institution would include the time needed to perform the confirmatory HIV-1/HIV-2 antibody differentiation assay (slightly less than 1 hour at CMJCVAMC per specimen) and the time needed to perform the viral load assay (about 6 hours to run a batch of 24 tests at CMJCVAMC). We calculated that confirmatory testing cost $\$ 184.51$ per test at CMJCVAMC. Replacing the third-generation assay with the more sensitive and specific fourth-generation test saved an estimated $\$ 3,875$ annually. This cost savings does not even consider savings in the pathologist/director's time for review- ing HIV results after the completion of the algorithm or the clinician/patient costs or anxiety while waiting for results of the confirmatory sequence of tests.

As diagnosis of HIV can have a significant psychological impact on the patient, it is important to ensure the diagnosis conveyed is correct. ${ }^{27}$ The provision of an HIV diagnosis to a patient has been described as a traumatic stressor capable of causing psychological harm; this harm should ideally be avoided if the HIV diagnosis is not accurate. There can be a temptation, when presented with a positive or reactive screening test that is known to come from an instrument or assay with a very high sensitivity and specificity, to present this result as a diagnosis to the patient. However, a false diagnosis from a false-positive screen would not only be harmful, but given the low prevalence of the disease in the screened population, would happen fairly frequently; in some settings the number of false positives may actually outnumber the number of true positive test results.

Better screening assays with greater specificity (even fractions of a percentage, given that specificities are already $>99 \%$ ) would help reduce the number of false positives and reduce the number of potential enticements to convey an incorrect diagnosis. Therefore, by adding an additional layer of safety through greater specificity, the fourthgeneration assay implementation helped improve the diagnostic safety of the laboratory and reduced the significant error risk to the clinician who would ultimately bear responsibility for conveying the HIV diagnoses to the patient. Given the increased prevalence of psychological and physical ailments in veterans, it may be even more important to ensure the diagnosis is correct to avoid increased psychological harm..$^{27,28}$

\section{Veteran Population}

For the general population, the fourthgeneration assay has been shown to be more sensitive and specific when compared with the third-generation assay due to the addition of detection of p24 antigen and the refinement of the antigenic targets for the antibody detection. ${ }^{6,8,11-13,18-20,22}$ However, the veteran population that receives VA medical care differs significantly from 
FIGURE 2 False-Positive Test Results

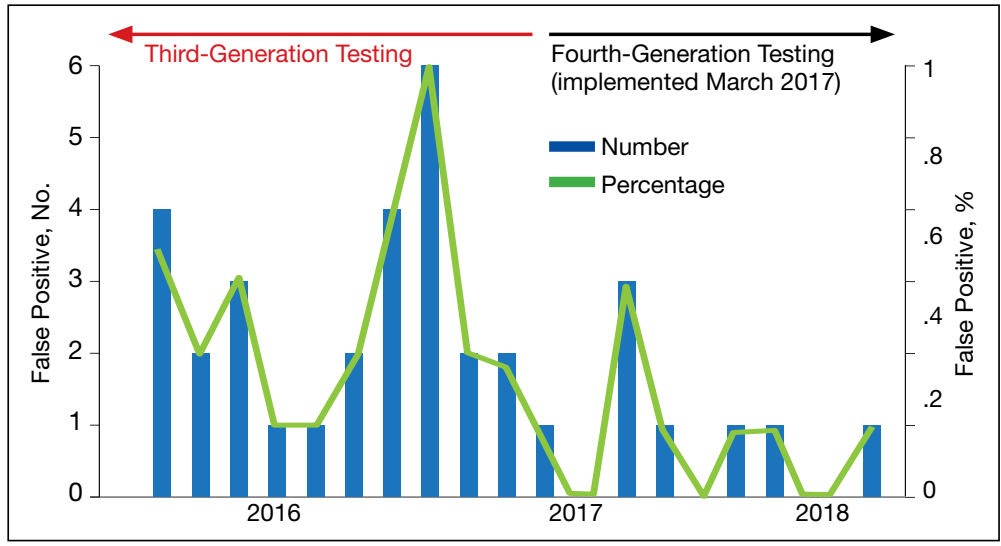

the nonveteran general population. Compared with nonveterans, veterans tend to have generally poorer health status, more comorbid conditions, and greater need to use medical resources. ${ }^{24-26}$ In addition, veterans also may differ in sociodemographic status, race, ethnicity, and gender. ${ }^{24-26}$

VA research in the veteran population is unique, and veterans who use VA health care services are an even more highly selected subpopulation. ${ }^{26}$ Conclusions made from studies of the general population may not always be applicable to the veteran population treated by VA health care services due to these population differences. Therefore, specific studies tailored to this special veteran population in the specific VA health care setting are essential to ensure that the results of the general population truly and definitively apply to the veteran population.

While the false-positive risk is most closely associated with testing in a population of low prevalence, it also should be noted that false-positive screening results also can occur in high-risk individuals, such as an individual on preexposure prophylaxis (PrEP) for continuous behavior that places the individual at high risk of HIV acquisition. ${ }^{8,29}$ The falsepositive result in these cases can lead to a conundrum for the clinician, and the differential diagnosis should consider both detection of very early infection as well as false positive. Interventions could include either stopping PrEP and treating for presumed early primary infection with HIV or continuing the PrEP. These interventions all have the potential to impact the patient whether through the production of resistant HIV virus due to the inadvertent provision of an inadequate treatment regimen, increased risk of infection if taken off PrEP as the patient may likely continue the behavior regardless, or the risks carried by the administration of additional antiretroviral therapies for the complete empiric therapy. Cases of an individual on PrEP who had a falsepositive HIV screening test has been reported previously both within and outside the veteran population. ${ }^{8}$ Better screening tests with greater sensitivity/specificity can only help in guiding better patient care.

\section{Limitations}

This quality assurance study was limited to retrospectively identifying the improvement in the false-positive rate on the transition from the third-generation to the more advanced fourth-generation HIV screen. False-positive screen cases could be easily picked up on review of the confirmatory testing per the CDC laboratory HIV testing algorithm. ${ }^{12,13}$ This study also was a retrospective review of clinically ordered and indicated testing; as a result, without confirmatory testing performed on all negative screen cases, a false-negative rate would not be calculable.

This study also was restricted to only the population being treated in a VA health care setting. This population is known to be different from the general population. ${ }^{24-26}$

\section{CONCLUSIONS}

The switch to a fourth-generation assay resulted in a significant reduction in falsepositive test results for veteran patients at CMJCVAMC. This reduction in falsepositive screening not only reduced laboratory workload due to the necessary confirmatory testing and subsequent review, but also saved costs for technologist's time and reagents. While this reduction in false-positive results has been documented in nonveteran populations, this is the first study specifically on a veteran population treated at a VAMC. ${ }^{8,11,18}$ This study confirms previously documented findings of improvement in the falsepositive rate of HIV screening tests with the change from third-generation to fourthgeneration assay for a veteran population. ${ }^{24}$ 
Author affiliations

Jeffrey Petersen and Sharvari Dalal are Staff Pathologists; Maria Monteiro is a Medical Technologist; and Darshana Jhala is the Chief of Pathology and Laboratory Medicine; all at the Department of Pathology and Laboratory Medicine, Corporal Michael J. Crescenz Veteran Affairs Medical Center. Jeffrey Petersen is an Assistant Professor of Clinical Pathology and Laboratory Medicine; Sharvari Dalal is an Adjunct Assistant Professor of Clinical Pathology and Laboratory Medicine; and Darshana Jhala is a Professor; all at the University of Pennsylvania Perelman School of Medicine in Philadelphia.

\section{Author disclosures}

The authors report no actual or potential conflicts of interest with regard to this article.

\section{Disclaimer}

The opinions expressed herein are those of the authors and do not necessarily reflect those of Federal Practitioner, Frontline Medical Communications Inc., the US Government, or any of its agencies.

\section{References}

1. Feinberg $M B$. Changing the natural history of HIV disease. Lancet. 1996;348(9022):239-246. doi:10.1016/s0140-6736(96)06231-9.

2. Alexander TS. Human immunodeficiency virus diagnostic testing: 30 years of evolution. Clin Vaccine $I m$ munol. 2016;23(4):249-253. Published 2016 Apr 4. doi:10.1128/CVI.00053-16

3. Mortimer PP, Parry JV, Mortimer JY. Which antiHTLV III/LAV assays for screening and confirmatory testing?. Lancet. 1985;2(8460):873-877. doi:10.1016/s0140-6736(85)90136-9

4. Holmberg SD, Palella FJ Jr, Lichtenstein KA, Havlir DV. The case for earlier treatment of HIV infection [published correction appears in Clin Infect Dis. 2004 Dec 15;39(12):1869]. Clin Infect Dis. 2004;39(11):1699-1704. doi:10.1086/425743

5. US Preventive Services Task Force, Owens DK, Davidson KW, et al. Screening for HIV Infection: US Preventive Services Task Force Recommendation Statement. JAMA. 2019;321(23):2326-2336. doi:10.1001/jama.2019.6587

6. Branson BM, Handsfield HH, Lampe MA, et al. Revised recommendations for HIV testing of adults, adolescents, and pregnant women in health-care settings. MMWR Recomm Rep. 2006;55(RR-14):1-CE4.

7. Bayer R, Philbin M, Remien RH. The end of written informed consent for HIV testing: not with a bang but a whimper. Am J Public Health. 2017;107(8):1259-1265. doi:10.2105/AJPH.2017.303819

8. Petersen J, Jhala D. Its not HIV! The pitfall of unconfirmed positive HIV screening assays. Abstract presented at: Annual Meeting Pennsylvania Association of Pathologists; April 14, 2018.

9. Wood RW, Dunphy C, Okita K, Swenson P. Two "HIV-infected" persons not really infected. Arch Intern Med. 2003;163(15):1857-1859. doi:10.1001/archinte.163.15.1857

10. Permpalung $N$, Ungprasert $P$, Chongnarungsin $D$, Okoli A, Hyman CL. A diagnostic blind spot: acute infectious mononucleosis or acute retroviral syndrome. Am J Med. 2013;126(9):e5-e6. doi:10.1016/j.amjmed.2013.03.017

11. Dalal S, Petersen J, Luta D, Jhala D. Third- to fourthgeneration HIV testing: reduction in false-positive results with the new way of testing, the Corporal Michael J. Crescenz Veteran Affairs Medical Center (CMCVAMC) Experience. Am J Clin Pathol.2018;150(suppl 1):S70-S71. doi:10.1093/ajcp/aqy093.172

12. Centers for Disease Control and Prevention. Laboratory testing for the diagnosis of HIV infection: updated recommendations. Published June 27, 2014. Accessed April 14, 2021. doi:10.15620/cdc.23447

13. Centers for Disease Control and Prevention. 2018 quick reference guide: recommended laboratory HIV testing algorithm for serum or plasma specimens. Updated January
2018. Accessed April 14, 202. https://stacks.cdc.gov/view /cdc/50872

14. Masciotra S, McDougal JS, Feldman J, Sprinkle P, Wesolowski L, Owen SM. Evaluation of an alternative HIV diagnostic algorithm using specimens from seroconversion panels and persons with established HIV infections. J Clin Virol. 2011;52(suppl 1):S17-S22. doi:10.1016/j.jcv.2011.09.011

15. Morton A. When lab tests lie ... heterophile antibodies. Aust Fam Physician. 2014;43(6):391-393.

16. Spencer DV, Nolte FS, Zhu Y. Heterophilic antibody interference causing false-positive rapid human immunodeficiency virus antibody testing. Clin Chim Acta. 2009;399(1-2):121-122. doi:10.1016/j.cca.2008.09.030

17. Kim S, Lee JH, Choi JY, Kim JM, Kim HS. False-positive rate of a "fourth-generation" HIV antigen/antibody combination assay in an area of low HIV prevalence. Clin Vaccine Immunol. 2010;17(10):1642-1644. doi:10.1128/CVI.00258-10

18. Muthukumar A, Alatoom A, Burns S, et al. Comparison of 4th-generation HIV antigen/antibody combination assay with 3rd-generation HIV antibody assays for the occurrence of false-positive and false-negative results. Lab Med. 2015;46(2):84-e29. doi:10.1309/LMM3X37NSWUCMVRS

19. Mitchell EO, Stewart G, Bajzik O, Ferret M, Bentsen C, Shriver MK. Performance comparison of the 4th generation Bio-Rad Laboratories GS HIV Combo Ag/Ab EIA on the EVOLIS TM automated system versus Abbott ARCHITECT HIV Ag/Ab Combo, Ortho Anti-HIV 1+2 EIA on Vitros ECi and Siemens HIV-1/O/2 enhanced on Advia Centaur. J Clin Virol. 2013;58(suppl 1):e79-e84. doi:10.1016/j.jcv.2013.08.009

20. Dubravac T, Gahan TF, Pentella MA. Use of the Abbott Architect HIV antigen/antibody assay in a low incidence population. J Clin Virol. 2013;58(suppl 1):e76-e78. doi:10.1016/j.jcv.2013.10.020

21. Montesinos I, Eykmans J, Delforge ML. Evaluation of the Bio-Rad Geenius HIV-1/2 test as a confirmatory assay. J Clin Virol. 2014;60(4):399-401. doi:10.1016/j.jcv.2014.04.025

22. van Binsbergen J, Siebelink A, Jacobs A, et al. Improved performance of seroconversion with a 4th generation HIV antigen/antibody assay. J Virol Methods. 1999;82(1):77-84. doi:10.1016/s0166-0934(99)00086-5

23. CLSI. User Protocol for Evaluation of Qualitative Test Performance: Approved Guideline. Second ed. EP12-A2. CLSI; 2008:1-46.

24. Agha Z, Lofgren RP, VanRuiswyk JV, Layde PM. Are patients at Veterans Affairs medical centers sicker? A comparative analysis of health status and medical resource use. Arch Intern Med. 2000;160(21):3252-3257. doi:10.1001/archinte.160.21.3252

25. Eibner C, Krull H, Brown KM, et al. Current and projected characteristics and unique health care needs of the patient population served by the Department of Veterans Affairs. Rand Health Q. 2016;5(4):13. Published 2016 May 9.

26. Morgan RO, Teal CR, Reddy SG, Ford ME, Ashton CM. Measurement in Veterans Affairs Health Services Research: veterans as a special population. Health Serv Res. 2005;40(5, pt 2):1573-1583. doi:10.1111/j.1475-6773.2005.00448.x

27. Nightingale VR, Sher TG, Hansen NB. The impact of receiving an HIV diagnosis and cognitive processing on psychological distress and posttraumatic growth. J Trauma Stress. 2010;23(4):452-460. doi:10.1002/jts.20554

28. Spelman JF, Hunt SC, Seal KH, Burgo-Black AL. Post deployment care for returning combat veterans. J Gen Intern Med. 2012;27(9):1200-1209. doi:10.1007/s11606-012-2061-1

29. Ndase P, Celum C, Kidoguchi L, et al. Frequency of false positive rapid HIV serologic tests in African men and women receiving PrEP for HIV prevention: implications for programmatic roll-out of biomedical interventions. PLOS One. 2015;10(4):e0123005. Published 2015 Apr 17. doi:10.1371/journal.pone.0123005 